\section{Case Reports in Oncology}

\title{
Hodgkin Lymphoma Mimicking Osteomyelitis
}

\author{
Aneela Majeed ${ }^{a, b} \quad$ Onyee Chan $^{b} \quad$ Onyemaechi Okolo $^{b}$ \\ Volodymyr Shponka ${ }^{c}$ Anca Georgescu ${ }^{a}$ Daniel Persky ${ }^{d}$ \\ ${ }^{a}$ Department of Infectious Diseases, University of Arizona, Banner University Medical \\ Center, Tucson, AZ, USA; ${ }^{b}$ Department of Medicine, University of Arizona, Banner \\ University Medical Center, Tucson, AZ, USA; ${ }^{\circ}$ Department of Pathology, University of \\ Arizona, Banner University Medical Center, Tucson, AZ, USA; ${ }^{d}$ Department of Hematology \\ Oncology, University of Arizona, Banner University Medical Center, Tucson, AZ, USA
}

\section{Keywords}

Hodgkin lymphoma · Lymphoma · Osteomyelitis · Osseous Hodgkin lymphoma

\begin{abstract}
Hodgkin lymphoma with symptomatic osseous involvement can have a similar presentation to osteomyelitis. Common findings in symptoms, laboratory workup, and imaging can make it very difficult to distinguish between the two diseases. Excisional biopsy should be pursued if fine-needle biopsy is equivocal and suspicion of lymphoma is high. We report a case of a 40-year-old man who presented with a history of marine animal sting on his neck and later developed erythema in the area, chest pain, constitutional symptoms, adenopathy, and imaging classic for sternal osteomyelitis. Fortunately, initial biopsy prompted the possibility of lymphoma, and further workup was initiated, which confirmed Hodgkin lymphoma. This case is a good reminder that malignancies and infections can share many common features, and keeping a broad differential diagnosis can be lifesaving. Proper staging and risk stratification of Hodgkin lymphoma help determine the optimal treatment.
\end{abstract}


 Oncology}

\section{Case Presentation}

A 40-year-old man presented after a presumed marine animal sting on his neck while scuba diving in Curacao 6 weeks ago, when a fire coral fish stung his neck, with a wheal forming immediately at the site of the lesion. On the following day, he also developed left axillary adenopathy and a rash on his center chest without vesicles or purulence. He was initially prescribed 2 weeks of cephalexin due to concern for possible cellulitis. With antibiotics, his adenopathy nearly resolved; however, the rash remained. Associated symptoms included chest pain that was both pleuritic and reproducible upon palpation, generalized myalgia, intermittent shortness of breath, and malaise.

Pertinent physical exam findings included a maculopapular rash overlying the sternum with severe tenderness to palpation and mild right axillary adenopathy. Laboratory workup was notable for an erythrocyte sedimentation rate (ESR) of $42 \mathrm{~mm} / \mathrm{h}$ and C-reactive protein of $4.08 \mathrm{mg} / \mathrm{dL}$. Complete blood count showed mild microcytic anemia with hemoglobin of $13.4 \mathrm{~g} / \mathrm{dL}$, normal white blood count of $9,400 / \mu \mathrm{L}$, and platelet count of $317,000 / \mu \mathrm{L}$. Computed tomography (CT) scan of the chest suggested an inflammatory process at the sternum with mediastinal involvement, so that osteomyelitis could not be ruled out. Subsequent magnetic resonance imaging of the sternum revealed diffuse T1 marrow replacement and corresponding enhancing edema involving the sternum, suggesting extensive osteomyelitis. There was also an area of nonenhancement in the manubrium, suggesting necrotic bone (Fig. 1a), along with extensive phlegmon in the muscles spanning $9 \mathrm{~cm} \times 5 \mathrm{~cm}$ (Fig. 1b) with multiple small abscesses and myositis.

The patient was taken to the operating room twice for extensive debridement of his left chest wall and was empirically started on vancomycin and cefepime. Wound culture eventually grew $1+$ Propionibacterium, and the patient was discharged on a 6-week course of vancomycin and Meropenem with the presumed diagnosis of osteomyelitis.

A few days after discharge, the patient's sternal bone biopsy from prior debridement came back with an atypical lymphohistiocytic infiltrate suggestive of Hodgkin lymphoma. Repeat CT of the chest showed multiple large bilateral axillary lymph nodes, and so ultrasound-guided right axillary lymph node biopsy was performed; however, the pathology only showed large atypical cells. Flow cytometry did not find any monotypic B-cell population, and therefore, it was not suggestive of a lymphoproliferative disorder. Given the high suspicion of lymphoma at this point, positron emission tomography (PET)/CT was done and located a highly fludeoxyglucose-avid right axillary lymph node measuring $2.4 \mathrm{~cm} \times 2.4 \mathrm{~cm}$ (Fig. 2a), in which excisional biopsy was performed. Pathology showed nodules of lymphocytes surrounded by fibrous bands, typical of classical Hodgkin lymphoma, nodular sclerosis variant (Fig. 3a). Reed-Sternberg cells and numerous lacunar cells were seen on a background of a mixed population of small lymphocytes (Fig. 3b). Immunohistochemistry revealed neoplastic cells positive for CD20, PAX5, CD30, and CD15, negative for CD45 and CD3, and Ki-67 positive in $40-50 \%$ of cells (Fig. $3 \mathrm{c}$, d). Together, this confirmed the diagnosis of nodular-sclerosis classical Hodgkin lymphoma.

Repeat PET/CT 3 weeks later showed an increase of fludeoxyglucose activity with a standardized uptake value from 3.7 to 24.9 on one of the index axillary nodes. The number and size of bilateral cervical and axillary adenopathy also increased (Fig. 2b) consistent with lymphoma progression. Due to discontinuous involvement of the sternum, even though there was no disease below the diaphragm, the patient was determined to have Hodgkin lymphoma stage IVA. His international prognostic score was 3. The patient was started on an AVD (doxorubicin, vinblastine, and dacarbazine) chemotherapy regimen. Bleomycin was not 


\section{Case Reports in Oncology}

added due to marginal diffusing capacity of the lung for carbon monoxide (DLCO). The plan was to add brentuximab later to the regimen.

\section{Discussion}

Hodgkin lymphoma is relatively rare with an estimated incidence rate of 2.6 cases per 100,000 people in the United States [1]. The nodular sclerosis subtype is more prevalent in young adults and often localized to the mediastinum, supraclavicular, and/or cervical lymph nodes $[2,3]$. Beyond the typical supradiaphragmatic adenopathy and constitutional symptoms, extranodal involvement is possible with common sites being the spleen, lungs, liver, and bone marrow [4]. Radiologic findings of occult osseous involvement can be found in up to $20 \%$ of cases; however, patients rarely present with symptomatic osseous lesions [5]. Ostrowski et al. [5] identified 25 cases of osseous Hodgkin disease over a span of 70 years at the Mayo Clinic. Vertebral involvement was most common (10 of 25), with pelvic bone closely following, and there were 3 cases of sternal involvement [5]. Many cases of osseous involvement were misdiagnosed as osteomyelitis owing to their similar initial presentations. In osteomyelitis, patients also commonly present with bone pain and nonspecific symptoms, such as fever, chills, and fatigue [6]. Both entities can be associated with the elevated inflammatory markers ESR and C-reactive protein. Radiologic evidence of osteolysis, osteosclerosis, mixed osteolysis/osteosclerosis, cortical destruction, and periosteal reaction are common in osseous Hodgkin lymphoma [5]. Imaging of the bone, regardless of the modality used, cannot distinguish Hodgkin lymphoma from osteomyelitis [7].

Reed-Sternberg cell within a reactive cellular background is pathognomonic for classical Hodgkin lymphoma [8]. Immunohistochemistry stain for these cells reveals positivity for CD30 and CD15. However, fine-needle aspiration or core-needle biopsy frequently are inadequate to make a reliable diagnosis and may show only nonspecific findings because of the low ratio of malignant cells and loss of architectural information [2]. When suspicion for lymphoma is high, further workup with excisional biopsy is crucial to help reach the correct diagnosis. Obviously, the treatment of osteomyelitis and Hodgkin lymphoma is completely different, and any delay in diagnosis is a disservice to the patient at best, if not detrimental.

Treatment for patients who are in an early stage (stage I-IIA) is guided by risk stratification. Patients with favorable prognostic features are treated with short-duration chemotherapy, typically 2 cycles of ABVD (doxorubicin, bleomycin, vinblastine, and dacarbazine) followed by 20 Gy restricted involved-field radiation therapy (IFRT) [2]. Those with unfavorable features, such as bulky mediastinal disease $(>10 \mathrm{~cm}$ greatest diameter), elevated ESR, and extranodal extension, are treated with a longer course of chemotherapy (4-6 cycles) followed by a higher dose of IFRT [2]. Patients with advanced stage (stage IIB-IV) are risk stratified by a different scoring system, namely the international prognostic score. Radiation in general is not beneficial in these patients. Our patient has stage IVA, and the plan is to provide more cycles of chemotherapy without IFRT.

\section{Conclusion}

In summary, our case illustrates the diagnostic difficulties between osteomyelitis and osseous Hodgkin lymphoma due to its rarity, similar presentations, and overlapping radiologic findings. If lymphoma is at all suspected, an excisional biopsy should be performed 
despite a negative fine-needle aspiration/biopsy. The importance of keeping a broad differential diagnosis even when all of the evidences are only pointing toward one disease initially cannot be overemphasized. In general, early-stage Hodgkin lymphoma is treated with a short course of chemotherapy followed by IFRT, whereas advanced-stage Hodgkin lymphoma is treated with a longer course of chemotherapy without IFRT.

\section{Acknowledgments}

We thank Dr. Faiz Anwer from the Department of Medicine - Hematology Oncology for critically reviewing this article and providing invaluable feedback.

\section{Statement of Ethics}

Verbal informed consent for publication of this patient's clinical details and images was obtained from the patient.

\section{Disclosure Statement}

The authors declare that there is no conflict of interest regarding the publication of this paper.

\section{Funding Sources}

There were no funding sources.

\section{References}

Siegel RL, Miller KD, Jemal A: Cancer statistics, 2016. CA Cancer J Clin 2016;66:7-30.

Ansell SM: Hodgkin lymphoma: 2016 update on diagnosis, risk-stratification, and management. Am J Hematol 2016;91:434-442.

-3 Eichenauer DA, Engert A, Andre M, Federico M, Illidge T, Hutchings M, et al: Hodgkin's lymphoma: ESMO Clinical Practice Guidelines for diagnosis, treatment and follow-up. Ann Oncol 2014;25(suppl 3):iii70-iii75.

4 Guermazi A, Brice P, de Kerviler EE, Ferme C, Hennequin C, Meignin V, et al: Extranodal Hodgkin disease: spectrum of disease. Radiographics 2001;21:161-179.

-5 Ostrowski ML, Inwards CY, Strickler JG, Witzig TE, Wenger DE, Unni KK: Osseous Hodgkin disease. Cancer 1999;85:1166-1178.

6 Lew DP, Waldvogel FA: Osteomyelitis. Lancet 2004;364:369-379.

-7 Bender Ignacio RA, Liu AY, Sohani AR, Vyas JM: Hodgkin's lymphoma masquerading as vertebral osteomyelitis in a man with diabetes: a case report. J Med Case Rep 2010;4:102.

8 Swerdlow SH, Campo E, Pileri SA, Harris NL, Stein H, Siebert R, et al: The 2016 revision of the World Health Organization classification of lymphoid neoplasms. Blood 2016;127:2375-2390. 


\section{Case Reports in Oncology}
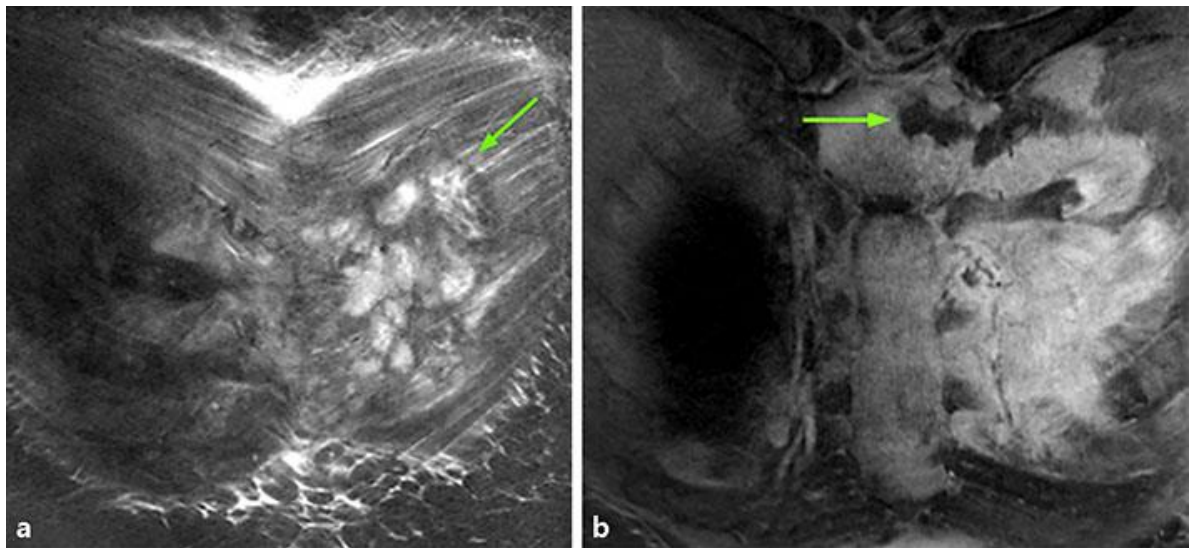

Fig. 1. T1-weighted magnetic resonance images of the sternum with and without contrast. a Extensive osteomyelitis of the manubrium and sternum body with an area of nonenhancement involving the left upper manubrium measuring $2.3 \mathrm{~cm} \times 1.5 \mathrm{~cm}$ (arrow) concerning for necrotic bone. $\mathbf{b}$ Extensive phlegmon involving the left first through fourth intercostal muscles and medial left pectoralis major spanning $9 \mathrm{~cm} \times$ $5 \mathrm{~cm}$ (arrow) with multiple small abscesses.
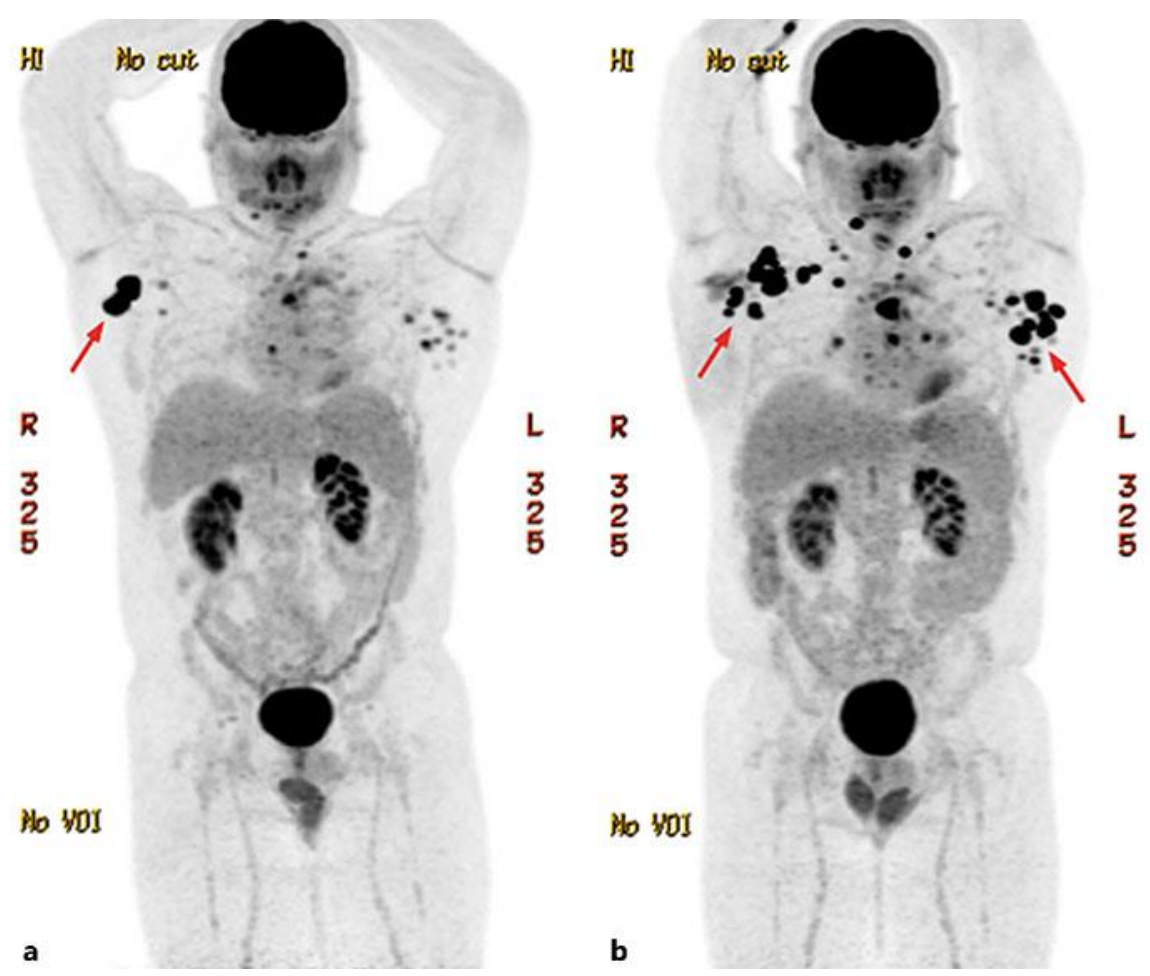

Fig. 2. Fludeoxyglucose (FDG) positron emission tomography (PET)/computed tomography (CT) from skull base to mid-thigh. a Multiple FDG-avid bilateral axillary lymph nodes are identified with the largest node on the right measuring $2.4 \mathrm{~cm} \times 2.4 \mathrm{~cm}$ (arrow), most likely reactive to osteomyelitis or an inflammatory process of the sternum. $\mathbf{b}$ Repeat PET/CT 3 weeks later showed progression of disease in terms of an increase in number, FDG activity, and size of bilateral cervical and axillary lymphadenopathy (arrows) consistent with lymphoma. 


\section{Case Reports in Oncology}
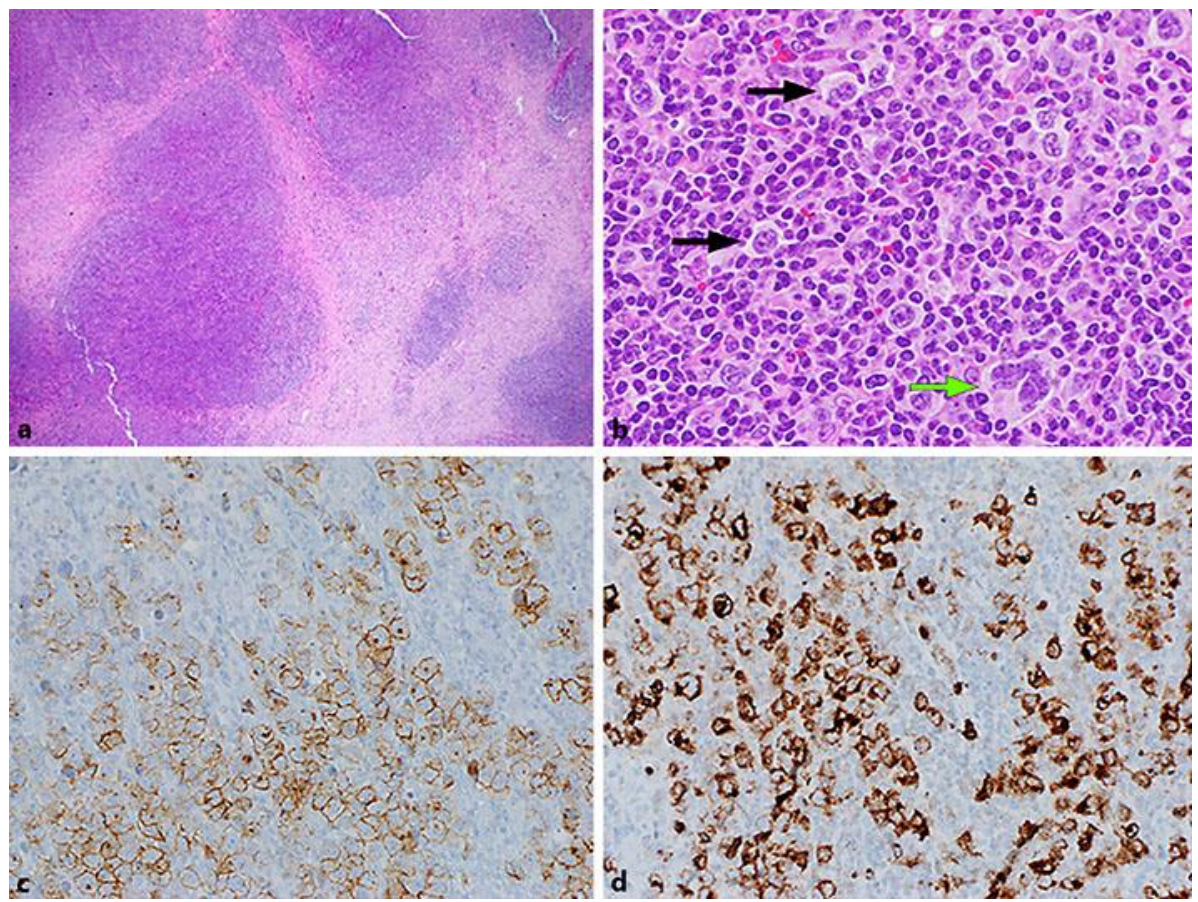

Fig. 3. Pathology of the right axillary lymph node. a HE stains of the specimen at $\times 20$ magnification demonstrating nodules of lymphocytes surrounded by fibrous bands, typical of classical Hodgkin lymphoma, nodular sclerosis variant. b Diagnostic Reed-Sternberg cell (green arrow) and numerous lacunar cells (black arrows) on a background of a mixed population of small lymphocytes. HE. $\times 400$. c Immunohistochemistry revealed positive staining of large neoplastic cells for CD30. $\mathbf{d}$ Positive staining for CD15. 